\title{
LA CAPACITACIÓN COMO ACCIÓN PREVIA EN UNA METODOLOGÍA PARA IMPLEMENTAR
} UN MANUAL DE PROCEDIMIENTOS TRAINING AS A PREVIOUS ACTION IN A METHODOLOGY TO IMPLEMENT A PROCEDURAL MANUAL

\author{
Daisy Hernández Corratge ${ }^{1}$. \\ Dra. Inidia Rubio Vargas².
}

1 E-mail: daysihc@ifal.uh.cu, Teléfono 72716110 Instituto de Farmacia y Alimentos de la Universidad de la Habana, Cuba. Daisy Hernández Corratge, cubana, es Lic. En Contabilidad y Finanzas en el año 2012, se ha desempeñado como Jefa del departamento de recursos humanos durante más de 15 años en el Instituto de Farmacia y Alimentos de la Universidad de la Habana. Las líneas de investigación desarrolladas han estado en el área de la dirección, gestión y liderazgo en la actividad educacional, actualmente es estudiante de postgrado de la Universidad Enrique José Varona, dentro del Programa Curricular Colaborativo en Dirección Educacional, especialidad Economía de la Educación donde se encuentra próxima a defender el grado científico de Dra. en Ciencias de la Educación, es miembro de Redipe desde diciembre del pasado año y se encuentra inscrita en ORCID con el código:0000-0003-1612-7252

2 E-mail: inidiarv@ifal.uh.cu; Teléfono 72020930. Instituto de Farmacia y Alimentos de la Universidad de la Habana. La Coronela. Cuba. Inidia Rubio Vargas, Cuba, Lic en Educación, especialidad Matemática, MsC. En Ciencias de la Educación y doctora en Pedagogía, es Profesora Titular Universidad de la Habana y profesora invitada de la Universidad de Ciencias Pedagógicas Enrique José Varona de la Habana. Coordinadora del Nodo RIDGE de la Red Iberoamericana de Pedagogía (Redipe) en Cuba. Coordinadora del proyecto MODELO DE GESTIÓN CON ENFOQUE DE PROCESOS DEL IFAL-UH (2017 -2019). Miembro del proyecto TEORÍA Y PRÁCTICA DE LAACTIVIDAD DE DIRECCIÓN EN LAS INSTITUCIONES EDUCATIVAS CUBANAS (2014 -2019). Miembro del comité académico del doctorado curricular colaborativo (2014-2019). Profesora Economía de la Educación. Miembro del comité académico de la especialización en Dirección Educativa en UCPEJV (2013-2019). Profesora Economía de la Educación. Ha realizado tutoría en el área de Pedagogía y Ciencias de la Educación en tesis de maestría y doctorados. Ha participado en eventos nacionales e internacionales, tales como: los congresos de Pedagogía, y Universidad, y Simposios Internacionales de REDIPE en Chile y Cuba. Presidenta Comité Organizador y Científico de Congresos, simposios y talleres internacionales; Primer Congreso de Alfabetización en Venezuela, 2009, Taller Ciencias Farmacéuticas y Alimentarias, Cuba, 2014, IV y V Simposio Redipe Cuba, junio y diciembre 2018.

Comité Científico II Simposio Internacional de Educación RIDGE. Dirección, Gestión, Liderazgo y Política Educativa. Universidad Católica del Muale. Chile.

Experiencia en la actividad de dirección como Vice Decana de las Facultades de Primaria y de Ciencias Exactas de la Universidad de Ciencias Pedagógicas Rafael M de Mendive, Jefa de Departamento Docente Matemática -Física en esta universidad y del Departamento Docente Formación general del IFAL - UH, Asesora planificación de la dirección de logística del Ministerio de Educación de la República de Cuba, actualmente es Asesora de la Directora General del IFAL-Uh y Jefa del grupo de Calidad. Tiene más de 50 publicaciones en libros y revistas indexadas y una amplia experiencia en la formación de Master y doctores. Se encuentra inscrita en ORCID con el código: 0000-0002-0525-3571. 
PALABRAS CLAVE: Capacitación, Manual de procedimientos, procesos de apoyo.

KEYWORDS: Training, procedures manual, support processes.

\section{RESUMEN}

EI IFAL en el contexto de las acciones que se desarrollan a nivel de país, del MES y de la Universidad de la Habana para la implementación de los Lineamientos de la Política Económica y Social del Partido y la Revolución, así como para perfeccionar la instrumentación del Sistema de Control Interno como método de gestión de la calidad orientado a la búsqueda de eficiencia, eficacia, pertinencia y competitividad institucional.

El presente trabajo es resultado de un proyecto de investigación que se desarrolla en el IFAL-UH desde 2017 "Modelo de gestión institucional con enfoque de procesos", la introducción de este modelo necesita la organización de un conjunto de acciones de capacitación que contribuya a la preparación de los actores de los diferentes procesos para el desempeño de las funciones que les corresponden respecto a las tareas institucionales.

Como ya se tiene el resultado parcial relativo al Manual de procedimientos de los procesos de apoyo el objetivo de este trabajo es fundamentar el programa de capacitación para la implementación del mencionado manual.

Última publicación:

2019. Inidia Rubio Vargas, Juan Abreu Payrol, Marian Hernández Colina. El enfoque de procesos, su concreción en un manual de gestión institucional. Libro de investigación. Educación y Pedagogía Cuba 2018. Parte V. Sello editorial REDIPE: ISBN: 978-1-945570-74-2

\section{ABSTRACT}

The IFAL in the context of the actions carried out at the country level, the MES and the University of Havana for the implementation of the Economic and Social Policy Guidelines of the Party and the Revolution, as well as to perfect the implementation of the System of internal control as a method of quality management aimed at the search for efficiency, effectiveness, relevance and institutional competitiveness. The present work is the result of a research project developed in the IFAL-UH since 2017. "Model of institutional management with a process approach", the introduction of this model requires the organization of a set of training actions that contribute to The preparation of the actors of the different processes for the performance of the functions that correspond to the institutional tasks.

As we already have the partial result related to the Procedures Manual of support processes, the objective of this work is to base the training program for the implementation of the mentioned manual.

\section{INTRODUCCIÓN}

Con el desarrollo exponencial de las comunicaciones y las tecnologías de la información, en esta sociedad del conocimiento y la información en la que vivimos, donde el conocimiento es poder y las sociedades más poderosas son aquellas donde se concentran esos conocimientos como resultado de la formación de conocimientos, habilidades y competencias en los recursos humanos, estos han llegado a ocupar el lugar más relevante en la ordenación jerárquica de los recursos institucionales.

Como expresión concreta de esta evolución se tiene que incluso los paradigmas de gestión en su modificación y enriquecimiento han llegado 
a evolucionar hoy a paradigmas centrados en los recursos humanos y su potencial creativo. (Gutiérrez, O, 2013).

Además, para las instituciones universitarias los recursos humanos son su potencial más preciado, puesto que son generadoras de conocimientos científicos, de cultura y de soluciones estratégicas que potencian el desarrollo sostenible, en el plano interno y externo o social, se remarca el papel que juegan los mismos en la eficiencia, calidad y pertinencia de los procesos universitarios.

Para el caso del Instituto de Farmacia y Alimentos de la Universidad de la Habana (IFAL-UH) dentro de las líneas de desarrollo estratégicas respecto a sus recursos humanos se distinguen acciones referidas a:

$\checkmark$ Perfeccionamiento de los indicadores bases para el cálculo de plantilla buscando mayor impacto de los profesores e investigadores en los procesos sustantivos y la actividad de gestión universitaria.

$\checkmark$ Sistematización de acciones de capacitación de todo el colectivo laboral incluyendo los que se desempeñan en los procesos de apoyo.

$\checkmark \quad$ Implementación de acciones para garantizar el tránsito de profesores desde las categorías inferiores hacia las categorías principales como resultado de su superación profesional, incluyendo el incremento de las escalas salariales teniendo esta base.

$\checkmark$ Estimulación a la formación de maestros en ciencias y doctores, apoyando estos procesos con la abertura de programas orientados a este fin, la introducción del pago por este concepto y las acciones para la utilización del potencial científico con máxima racionalidad.

El presente trabajo es resultado de un proyecto de investigación que se desarrolla en el IFAL-UH desde 2017 "Modelo de gestión institucional con enfoque de procesos", Rubio, I, 2017, la introducción de este modelo necesita la organización de un conjunto de acciones de capacitación que contribuya a la preparación de los actores de los diferentes procesos para el desempeño de las funciones que les corresponden respecto a las tareas institucionales.

Como ya se tiene el resultado parcial relativo al Manual de procedimientos de los procesos de apoyo el objetivo de este trabajo es fundamentar el programa de capacitación para la implementación del mencionado manual.

\section{MÉTODOS UTILIZADOS}

Modelación: utilizado en el proceso de determinación de los componentes del programa de capacitación y el establecimiento de las relaciones entre estos

Análisis del producto de la actividad, encuestas de autovaloración para realizar el levantamiento diagnóstico de necesidades de superación.

\section{RESULTADOS}

\section{Fundamentos del programa de superación}

El IFAL en el contexto de las acciones que se desarrollan a nivel de país, del MES y de la Universidad de la Habana para la implementación de los Lineamientos de la Política Económica y Social del Partido y la Revolución, así como para perfeccionar la instrumentación del Sistema de Control Interno como método de gestión de la calidad orientado a la búsqueda de eficiencia, eficacia, pertinencia y competitividad institucional, ha estado gestionando el cambio de sus directivos y trabajadores en los modelos de dirección, los métodos y estilos, buscando una visión más óptima de las relaciones entre los procesos y subprocesos institucionales, 
las funciones de los agentes y agencias en los diferentes niveles de dirección.

Según las bases normativas tanto del Sistema de Control Interno como de la gestión de la calidad se encuentra la procedimentación de las tareas por procesos y subprocesos, ya que es la base para el incremento y sostenibilidad de los resultados (en indicadores) tanto de los procesos como de los niveles de desarrollo de la cultura organizacional y la implementación de Manuales de procedimientos en los diferentes procesos como herramienta para su gestión.

En los procesos de autocontrol de los cuatro últimos cursos, reuniones de trabajo de la Alta Dirección, del Consejo de Dirección, de las Comisiones de trabajo permanente: Comisión Consultiva de Cuadros, Comité de Prevención y Control de Riesgos, Planificación Estratégica y la comisión temporal para la elaboración de los procedimientos, los manuales y su instrumentación, se ha identificado la necesidad de incrementar las acciones de preparación a los cuadros y trabajadores como parte de la gestión del cambio en la actividad de dirección. En particular, el IFAL está organizándose en una nueva estructura que marca diferencias significativas con las anteriores, para alcanzar mayor coherencia y eficiencia de la actividad pedagógica profesional del claustro de carrera, de los procesos de investigación, gestión del conocimiento, de la gestión administrativa y sus procesos de apoyo, para que esta desde las tareas de los trabajadores cumpla su misión institucional.

Dentro de las acciones que se ejecutan se encuentra el diseño, implementación y mejoramiento del conjunto de procedimientos para el área administrativa, desde noviembre de 2017 la Comisión de Planificación Estratégica supervisada por el Comité de Prevención y Control del IFAL-UH, ha estado realizando la tarea de procedimentación para el área administrativa y en el diagnóstico inicial se identifican problemáticas de preparación para la implementación de estos procedimientos.

Este programa está dirigido a buscar niveles de transformación más inmediato en la actividad de dirección y el ajuste a las necesidades y particularidades de los directivos y funcionarios que se desempeñan en la Dirección Administrativa, así como de todos los implicados en la actividad de procedimentación.

\section{PARTICULARIDADES DE LA DIRECCIÓN ADMINISTRATIVA COMO SUJETOS DEL PROGRAMA DE SUPERACIÓN.}

El funcionamiento de una institución tiene diversas variables que condicionan y hasta determinan su organización y junto a esta, su imagen interna y externa, las condiciones de estudio, trabajo y vida; máxime cuando se trata de una institución formadora de profesionales universitarios que necesita de un soporte logístico - material para el cumplimiento con la mayor pertinencia posible de su misión en correspondencia con las características actuales de las universidades cubanas.

Todo intento orientado al mejoramiento de la gestión tiene que implicar no solamente los procesos sustantivos: formación de pregrado y postgrado; investigación, innovación y producción; extensión universitaria, puesto que se requiere del perfeccionamiento tanto de los procesos orientados al crecimiento y desarrollo estratégico como de aquellos procesos de apoyo que se encuentran indirectamente relacionados con la misión.

En el marco del perfeccionamiento continuo del sistema de educación superior cubano, se han transitado por diversas etapas marcadas no solamente por la concepción pedagógica de la formación, sino también por la mirada económica que necesitan las instituciones adscriptas a este sistema; este enfoque no es solamente cubano, a nivel mundial la gestión se ha reorientado 
también hacia cómo incrementar los indicadores atinentes a la eficiencia y la eficacia.

Para nuestro caso las acciones han respondido a las características de nuestro modelo de desarrollo económico y social, donde garantizar los niveles de la actividad de la Educación Superior ha sido una prioridad en la asignación de presupuestos del estado, incluso en los años difíciles del llamado Período Especial.

El entorno actual requiere entonces que tanto los directivos como los trabajadores hagan un uso más racional de los recursos humanos, materiales y financieros, el incremento sostenible del control participativo y estratégico sobre estos, el cuidado y la protección de los bienes que son patrimonio institucional, la focalización de los procesos claves para gestionar los riesgos vistos estos tanto desde sus manifestaciones negativas para minimizarlas, como también desde las oportunidades que pueden representar para la necesaria apertura y entrelazamiento con el contexto.

Sobre uno de estos procesos se ha estado avanzando desde finales del año 2017 , teniendo como referentes inmediatos los resultados que se han ido alcanzando en términos de planificación estratégica e implementación del Sistema de Control Interno, los cuales aunque no han sido sistemáticos si han constituido base para esta etapa de trabajo, que por primera vez toma como centro de atención los antes mencionados procesos de apoyo, en particular los que se gestionan desde la Dirección Administrativa.

\section{¿QUÉ ES LA DIRECCIÓN ADMINISTRATIVA DEL INSTITUTO DE FARMACIA $Y$ ALIMENTOS?}

Formalmente en el organigrama de la institución, esta es un área de subordinación directa al decanato - dirección, cuya misión es la gestión de los recursos materiales y de los servicios que garantizan el funcionamiento de todos los procesos institucionales con un enfoque de mejoramiento continuo e incremento de los niveles de satisfacción de estudiantes y profesores.

En la estructura aprobada a finales del año 2017 la Dirección Administrativa se organiza según el esquema que sigue:
Esta dirección tiene nombrado un Director Administrativo que es un cuadro de dirección y cada grupo de trabajo tiene nombrado un funcionario que se desempeña como Jefe de Grupo con un conjunto de tareas que se han actualizado como resultado de este trabajo y que se presentan en este manual de procedimientos.

El proceso de ejecución de tareas y solución de problemas del área involucra a actores de los 
diferentes grupos, desde el cumplimiento de las funciones de dirección se establecen relaciones de complementación, ejecución conjunta, control y comunicación e información que requieren su gestión desde el Director Administrativo.

Estos elementos que caracterizan a la dirección administrativa como proceso de apoyo, condicionan la determinación de los componentes del programa de superación.

\section{OBJETIVOS}

1. Argumentar los aspectos distintivos del modelo de gestión por procesos, la gestión de la calidad y su relación con la implementación del Sistema de Control Interno.

2. Valorar el conjunto de acciones directivas para planificar, organizar, regular y controlar el proceso de implementación del Manual de procedimientos, particularizando en la DA dentro del sistema de trabajo del IFAL, considerando sus particularidades y la nueva estructura aprobada en fecha reciente.

\section{TEMAS Y SU CONTENIDO}

Tema 1: La dirección como proceso y sus funciones. Caracterización del sistema de trabajo del IFAL desde los manuales de organización, funcionamiento y de procedimientos. El Sistema de Control Interno como método de gestión institucional. La gestión de la calidad, el enfoque integrado del SCl y la Gestión de la calidad. La procedimentación como un núcleo articulador.

Tema 2: Estructura y sistema de trabajo desde la DA. Las tareas fundamentales de los procesos de apoyo y los procedimientos para ejecutarlas. El enfoque de competencias en la gestión institucional. La dirección del trabajo en equipo como premisa para elevar la calidad de la ejecución y control de las tareas procedimentadas.

\section{Tema 3:}

La procedimentación. Metodología y su enfoque para el caso particular IFAL-UH. Tareas de los actores que ejecutan la procedimentación. Tareas de los actores que controlan y supervisan la implementación de los procedimientos. Estudio de casos en grupos de trabajo de la DA como proceso de apoyo.

\section{HABILIDADES}

- Resolver problemas profesionales de dirección en el nuevo contexto de organización de la DA y el enfoque de la dirección por procesos.

- Organizar el sistema de acciones de los actores para ejecutar la actividad de procedimentación. 
Tabla 1: Cronograma de actividades

\begin{tabular}{|c|c|c|c|c|}
\hline No & $\begin{array}{l}\text { Tipo de } \\
\text { actividad }\end{array}$ & Contenido & $\begin{array}{c}\text { Horas } \\
\text { presenciales }\end{array}$ & $\begin{array}{c}\text { Horas no } \\
\text { presenciales }\end{array}$ \\
\hline 1 & СT & $\begin{array}{l}\text { La dirección como proceso y sus funciones. El } \\
\text { Sistema de Control Interno como método de } \\
\text { gestión institucional. La gestión de la calidad, } \\
\text { el enfoque integrado del } \mathrm{SCl} \text { y la Gestión de la } \\
\text { calidad. La procedimentación como un núcleo } \\
\text { articulador. }\end{array}$ & $4 \mathrm{~h}$ & $12 \mathrm{~h}$ \\
\hline 2 & Taller & $\begin{array}{l}\text { Caracterización del sistema de trabajo del } \\
\text { IFAL desde los manuales de organización, } \\
\text { funcionamiento y de procedimientos. }\end{array}$ & $4 \mathrm{~h}$ & $12 \mathrm{~h}$ \\
\hline 3 & CT & $\begin{array}{l}\text { Estructura y sistema de trabajo desde la DA. Las } \\
\text { tareas fundamentales de los procesos de apoyo } \\
\text { y los procedimientos para ejecutarlas. El enfoque } \\
\text { de competencias en la gestión institucional. La } \\
\text { dirección del trabajo en equipo como premisa } \\
\text { para elevar la calidad de la ejecución y control de } \\
\text { las tareas procedimentadas. }\end{array}$ & $4 \mathrm{~h}$ & $2 \mathrm{~h}$ \\
\hline 4 & Taller & Estudios de casos procedimientos diseñados DA & $4 \mathrm{~h}$ & $8 \mathrm{~h}$ \\
\hline 5 & CT & $\begin{array}{l}\text { La procedimentación. Metodología y su enfoque } \\
\text { para el caso particular IFAL-UH. Tareas de } \\
\text { los actores que ejecutan la procedimentación. } \\
\text { Tareas de los actores que controlan y supervisan } \\
\text { la implementación de los procedimientos. Estudio } \\
\text { de casos en grupos de trabajo de la DA. }\end{array}$ & $4 \mathrm{~h}$ & $4 \mathrm{~h}$ \\
\hline 6 & Taller & $\begin{array}{l}\text { Estudio de casos implementación de los } \\
\text { procedimientos diseñados DA. }\end{array}$ & $4 \mathrm{~h}$ & $12 \mathrm{~h}$ \\
\hline 7 & $\begin{array}{l}\text { Taller } \\
\text { Final }\end{array}$ & $\begin{array}{l}\text { Planificación del entrenamiento metodológico } \\
\text { en el puesto de trabajo para los directivos } \\
\text { y funcionarios de la DA. Valoración final. } \\
\text { Conclusiones. }\end{array}$ & $4 \mathrm{~h}$ & $2 \mathrm{~h}$ \\
\hline
\end{tabular}

De la tabla 1 se calculan las horas totales del programa y por tipo de actividad de aprendizaje:

Total de horas: 70

Encuentros presenciales: $7,28 \mathrm{~h}$ 
Trabajo independiente: 52 horas

Conferencias talleres: $12 \mathrm{~h} \quad$ Talleres: $16 \mathrm{~h}$

Tabla 2: Distribución por semanas:

\begin{tabular}{|l|l|}
\hline Semana & Actividad \\
\hline 12 al 16 de marzo & Actividad 1, CT \\
\hline 19 al 23 de marzo & Actividad 2, Taller 1 \\
\hline 26 al 30 de marzo & Actividad 3, CT \\
\hline 2 al 6 de abril & Actividad 4, Taller 2 \\
\hline 9 al 13 de abril & Actividad 5, CT \\
\hline 23 al 27 de abril & Actividad 6, Taller 3 \\
\hline 30 abril al 4 de mayo & Actividad 7, TF \\
\hline
\end{tabular}

La tabla 2 muestra la distribución de actividades por períodos de tiempo, una vez concluido este período de alrededor de dos meses se continuan las acciones de preparación a través del adiestramiento en el puesto de trabajo.

\section{LA CONCEPCIÓN DEL ADIESTRAMIENTO EN EL PUESTO DE TRABAJO COMO PARTE DEL PROGRAMA DE SUPERACIÓN.}

Esta concepción parte de los fundamentos generales del proyecto que se han socializado en trabajos de Rubio, et al (2017), (2018) enmarcados en los procesos de gestión del conocimiento y gestión de la calidad donde se identifica la gestión individual del conocimiento como parte del capital estructural de la institución para transformarlo en activo intangible impactando la calidad de los diferentes procesos institucionales.

\section{OBJETIVO:}

capacitar a los directivos y funcionarios del área de la DA para implementar, mejorar y perfeccionar los procedimientos en el proceso de ejecución de sus tareas.
Las tareas que se identifican son las siguientes:

1. Preparación previa a los directivos y funcionarios del área de la DA y a los actores que procedimentan. (curso de superación)

2. Diagnóstico-caracterización del colectivo de trabajadores y del equipo de dirección de la DA.

3. Proyección del sistema de trabajo para la implementación de los procedimientos desde los planes mensuales-individuales y la organización del trabajo en equipos.

4. Seguimiento a la implementación del sistema de trabajo en la atención individual.

5. Evaluación integral del entrenamiento y presentación del informe resumen al CPC.

\section{METODOLOGÍA}

PARA

EL

\section{ENTRENAMIENTO:}

Primero: selección del equipo de entrenadores. Segundo: preparación previa del equipo de entrenadores

a) Concentrada: de conjunto con los demás actores.

b) Sistemática: previa al inicio de cada tarea, salida al plan de trabajo.

Tercero: ejecución de las acciones de entrenamiento en el puesto de trabajo.

Valoración de la transformación del desempeño en la actividad de dirección: reunión conjunta con el equipo de dirección DA.

Cuarto: Elaboración del informe final, las conclusiones y recomendaciones en reunión de todo el equipo. Plan de seguimiento y control al desarrollo de la actividad de dirección en los departamentos. 
BIBLIOGRAFÍA PARA EL PROGRAMA:

$\checkmark$ Manuales de organización, funcionamiento y procedimientos del IFAL-UH

$\checkmark$ Resolución 60/11

$\checkmark$ Resolución 210/07

$\checkmark$ Documentos del MES para perfeccionar la gestión en el nivel de base

$\checkmark$ Manual de Procedimientos DA (en construcción)

\section{RESULTADOS OBTENIDOS EN EL DESARROLLO DEL PROGRAMA DE SUPERACIÓN.}

Al finalizar el programa de superación se aplica la técnica del PNI para valorar los aspectos positivos, negativos e interesantes como acción para la mejora del mismo, lo cual es además un referente para las acciones a realizar en el resto de los procesos, a continuación y a modo de cierre se presenta un resumen de los planteamientos de los sujetos participantes:

\section{ASPECTOS POSITIVOS SEÑALADOS:}

$\checkmark$ La posibilidad de auto valorar el nivel de preparación que se tiene para la implementación del Manual de procedimientos y comparar respecto a otros participantes.

$\checkmark$ Conocer los procedimientos que se van a implementar e intercambiar con actores de otras áreas que ejecutan acciones en los procedimientos.

$\checkmark$ Preparase para introducir el modelo de gestión con enfoque de proceso desde las funciones que se enriquecen por las relaciones de horizontalidad.

$\checkmark \quad$ La posibilidad de intercambiar y unificar estrategias de trabajo en los distintos grupos de trabajo. $\checkmark$ Que la institución organice acciones de superación a actores de procesos de apoyo poniendo énfasis en la relevancia de estos para todos los procesos.

$\checkmark$ Explicación concreta y didáctica de los temas e incremento de los conocimientos relativos a la gestión y a otros temas de actualidad.

$\checkmark$ Herramientas necesarias para organizar el trabajo en equipos multidisciplinares, el liderazgo y las relaciones entre los modelos de gestión desde los procedimientos.

\section{ASPECTOS NEGATIVOS:}

$\checkmark$ Coincidencia del curso con otras actividades originando ausencias.

$\checkmark$ Podría ser más extenso, faltó más tiempo para sistematizar lo aprendido.

$\checkmark \quad$ La no participación de todos los actores de los procesos.

$\checkmark \quad$ La duplicación hacia la base que necesita inmediatez

$\checkmark$ Permanencia de los compañeros en la preparación, a veces tenían que salir antes de tiempo por la dinámica de los procesos de apoyo.

\section{ASPECTOS INTERESANTES:}

$\checkmark$ Todos los aspectos

$\checkmark$ Poder compartir la experiencia de profesionales en el tema, que de una u otra forma pudiéramos ser los mismos que nos encontremos al enfrentar esta gran tarea.

$\checkmark \quad$ Potenciar el uso de variantes que posibiliten mejorar la organización de los actores en los procesos de apoyo 
$\checkmark$ Método utilizado por el colectivo de entrenadores.

$\checkmark \quad$ La forma en que se organizó el contenido que permite aplicarlo en la práctica

$\checkmark \quad$ La transformación de las funciones de los actores desde la dirección institucional como resultado de la implementación del Manual de procedimientos.

\section{CONCLUSIONES}

La superación de los actores es una necesidad ya la vez constituye premisa para la implementación de un Manual de procedimientos, ya que este es resultante de un modelo de gestión con enfoque de procesos que difiere del modelo actuante estructura - función, dentro de la preparación para el cambio es además una acción para la sensibilización de todos los implicados.

Los procesos de apoyo que se gestionan desde la Dirección Administrativa del IFAL-UH requieren de la procedimentación de tareas comenzando por aquellas donde se concentran más riesgos, la implementación del Manual de procedimientos contribuye a perfeccionar la ejecución de las actividades, pero requiere de una preparación que permita a todos los actores entender las nuevas funciones y el ordenamiento interno de la secuencia de actividades que conforma cada procedimiento.

\section{REFERENCIAS BIBLIOGRÁFICAS}

Lineamientos de la Política Económica y Social de la Revolución, 2011; Conceptualización del Modelo Económico Cubano, 2017; Ejes de desarrollo estratégico hasta el 2030, 2017.

Normas ISO, 9000/2005; 9001/2015; 9004/2018, Red Internacional de Aseguramiento de la Calidad de la Educación Superior (INQAAHE, 2007)
Orientaciones de buenas prácticas (RIACES (Red Iberoamericana para la Acreditación de la Calidad de la Educación Superior)

Prietula y Simón, 1989; Jones y Wright Barney 1991; 1992; Adam Smith, Theodore W. Schultz y Gary Becker 1993; Wright y Mcmaham, 1992; Bailey, 1993; Huselid, 1995; Brooking 1996; Edvisson y Malone 1997; Stewart 1998; Ulrich, 1998; Gutiérrez, 2001, 2003, 2009,2013; Sallenave, 2001; Andrade, 2005

Ramírez, J. 2013. La integración del sistema de control interno y el sistema de gestión de la calidad: una opción de mejora en la empresa. Editorial Centro de Estudios de la Economía Cubana. La Habana. Cuba.

Rubio, I, Abreu J, Hernández M. EL PROCESO DE GESTIÓN DEL CONOCIMIENTO; UNA VISIÓN ESTRATÉGICA PARA EL IFALUH. Revista de Ciencias Farmacéuticas y Alimentarias. Volumen 4, No 1, ISSN: 2411927X. Grupo 4.

Rubio, I, Abreu J, Hernández M. La gestión de la calidad en la Universidad actual, una visión estratégica para el caso IFAL-UH. Revista Boletín REDIPE. Volumen 4. No 38. ISBN: 9781-945570-50-6. Grupo 3.

Rubio, I, Abreu J, Hernández M. Tres perspectivas de la formación del conocimiento en la gestión universitaria. Revista Boletín REDIPE. Volumen 4. No 38. ISBN: 978-1-945570-38-4. Grupo 3.

Rubio, I, Abreu J, Martínez, M. 2017. Modelo de gestión de la institución universitaria con enfoque de procesos. Libro Dirección, Gestión, Liderazgo y Política Educativa. ISBN: 978-$\square$ 958-- $\square$ 8967-- $\square 20--\square 2$ Sello Editorial REDIPE (95857440). Red de Pedagogía S.A.S. NIT: 900460139-2. Colombia. 
Rubio, I, Abreu, J, Hernández, M, 2019. El enfoque de procesos, su concreción en un manual de gestión institucional. . Libro de investigación. Educación y Pedagogía Cuba 2018. Parte V. Sello editorial REDIPE: ISBN: 978-1-945570-74-2.

Sánchez, M, 2005. Breve inventario de los modelos para la gestión del conocimiento en las organizaciones

Sistema de evaluación y acreditación de Instituciones Educación Superior (SEA-IES). Junta de acreditación nacional. 2014, Resolución 77/2018 JAN. 